\title{
Wide-field absolute transverse blood flow velocity mapping in vessel centerline
}

Nanshou $\mathrm{Wu}$

Lei Wang

Bifeng Zhu

Caizhong Guan

Mingyi Wang

Dingan Han

Haishu Tan

Yaguang Zeng 


\title{
Wide-field absolute transverse blood flow velocity mapping in vessel centerline
}

\author{
Nanshou Wu, Lei Wang, Bifeng Zhu, Caizhong Guan, Mingyi Wang, Dingan Han, Haishu Tan, and \\ Yaguang Zeng*, \\ Foshan University, Department of Physics and Optoelectronic Engineering, Guangdong, China
}

\begin{abstract}
We propose a wide-field absolute transverse blood flow velocity measurement method in vessel centerline based on absorption intensity fluctuation modulation effect. The difference between the light absorption capacities of red blood cells and background tissue under low-coherence illumination is utilized to realize the instantaneous and average wide-field optical angiography images. The absolute fuzzy connection algorithm is used for vessel centerline extraction from the average wide-field optical angiography. The absolute transverse velocity in the vessel centerline is then measured by a cross-correlation analysis according to instantaneous modulation depth signal. The proposed method promises to contribute to the treatment of diseases, such as those related to anemia or thrombosis. @ 2018 Society of Photo-Optical Instrumentation Engineers (SPIE) [DOI: 10.1117/1.JBO.23.2 .026008]
\end{abstract}

Keywords: wide-field; absolute transverse blood flow velocity; vessel centerline.

Paper 170568RR received Aug. 29, 2017; accepted for publication Feb. 5, 2018; published online Feb. 27, 2018.

\section{Introduction}

Determination of blood flow velocity is important in the study of ophthalmic and brain diseases. ${ }^{1,2}$ Eye and brain blood flow velocities are particularly important health indicators. Some optical imaging technologies have been developed for blood flow velocity measurement, such as laser speckle imaging ${ }^{3,4}$ and laser Doppler imaging. ${ }^{5}$ These two methods are based on the Doppler effect. The measurement of blood flow velocity based on the Doppler effect is affected by the Doppler angle between the laser beam and the direction of flow at the flow sampling site. However, the traditional Doppler method cannot provide an accurate blood flow velocity measurement because the angle of the vessel is not accurately obtained in practical applications. The bidirectional laser Doppler velocimetry ${ }^{2,6-8}$ has been developed to obtain an accurate blood flow velocity measurement. However, it further increases the complexity of the system. In addition, the related literature ${ }^{9}$ proposes a method of single blood cell imaging using adaptive optics scanning light ophthalmoscope in the absence of contrast agent in the eyes of living mice. The method obtains the average blood flow velocity of capillary by calculating the average speed of all red blood cells (RBCs) in capillaries. This method of calculating blood flow velocity is viable in a few capillaries, but it is not feasible in larger area vessels. In addition, due to the complexity and cost of the adaptive optics system, its application is limited. For some flat biological samples, such as chicken embryos, zebra fish, and retinas, the imaging plane is perpendicular to the incident light direction. Hence, the blood flow motion is transverse, thus necessitating a new method to trace the RBCs to perform an absolute transverse flow velocity (ATFV) measurement.

Another practical problem is that according to fluid mechanics, the flow velocity of a fluid through a pipe tends to be higher at the middle compared with that close to the sides of the pipe. ${ }^{10}$ A blood vessel is a kind of small pipe, and the flow velocity distribution in it exhibits this fluid flow characteristic. Hence, the ATFV along the centerline of a vessel is more representative of the overall ATFV, and the study of the ATFV along vessel centerlines is thus of utmost significance. Based on the above analysis, the measurement of ATFV involves two key challenges. First, the movement of RBCs needs to be tracked and to be distinguished from the background tissue. Second, the displacement of the vessel centerline and the transition time of the RBCs in two related positions need to be measured.

In the present study, we achieved wide-field centerline ATFV measurement in a vessel based on the absorption intensity fluctuation modulation (AIFM) effect. ${ }^{11}$ Because of the difference in absorption between the RBCs and the background tissue under low-coherence light illumination at a center wavelength of $540 \mathrm{~nm},{ }^{12}$ an endogenous instantaneous intensity fluctuation is generated by the AIFM effect when RBCs discontinuously traverse the blood vessel. The AIFM effect is used to obtain the wide-field optical angiography image and to highlight the RBCs' signal relative to the background tissue by computing the average modulation depth (AMD) and the instantaneous modulation depth (IMD). The vessel centerline can be subsequently determined from the AMD image. The ATFV along the vessel centerline is finally determined from the high signalto-noise ratio (SNR) IMD sequence image according to the formula $\nu=\Delta L / \Delta \tau_{A B}$, where $\nu$ is the ATFV along the vessel centerline, and $\Delta \tau_{A B}$ and $\Delta L$ are the transition time and relative displacement between the two cross-correlated locations $A$ and $B$ along the vessel centerline, respectively.

\section{Experimental Setup and Materials}

As shown in Fig. 1(a), the low-coherence light source was a fiber-coupled light-emitting diode source $\left(\lambda_{0}=540 \mathrm{~nm}\right.$, bandwidth $=10 \mathrm{~nm}$, power $=100 \mathrm{~mW}$ ). The light was divided into four collimated beams by a $1 \times 4$ fiber splitter and passed 

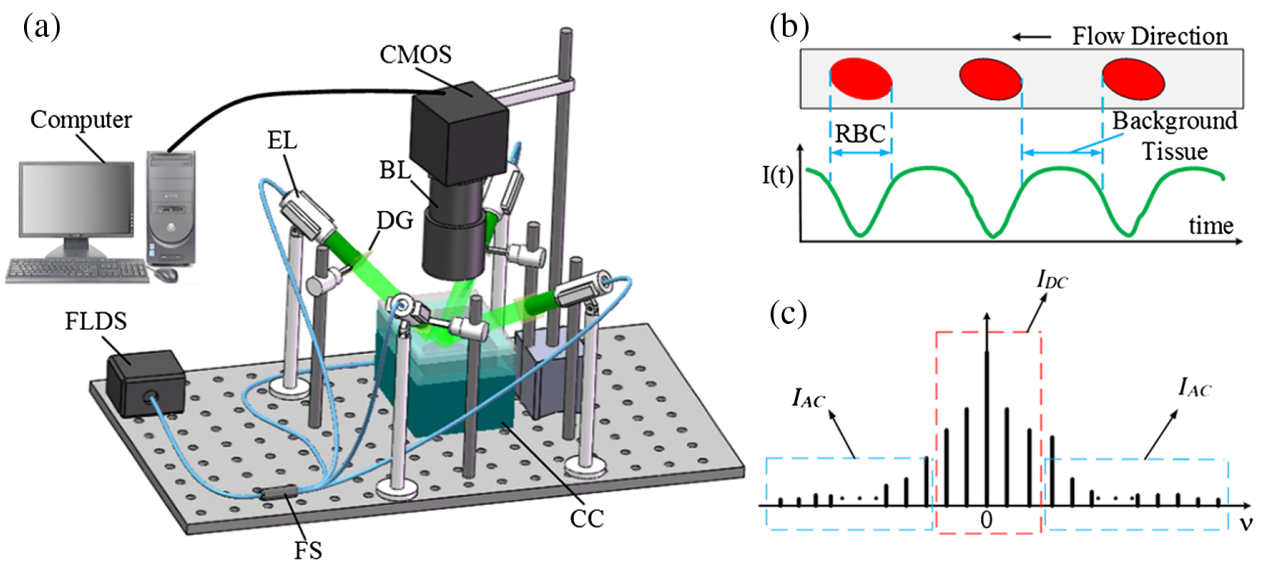

Fig. 1 (a) Experimental setup, (b) AIFM effect, and (c) separation of the RBCs' signal from that of the background tissue.

through an extended lens and a diffusing glass, and was then used to illuminate the experimental samples. The biological samples comprised chicken embryos placed in a custom container at a constant temperature of $37.8^{\circ} \mathrm{C}$ and $75 \%$ humidity. The diffuse light was propagated through a zoomed bitelecentric lens (the depth of focus $0.5 \mathrm{~mm}$ ) to a high-speed complementary metal oxide semiconductor camera (acA 2040-180 km, Basler, Germany). The actual pixel size of the camera was $5.5 \times 5.5 \mu \mathrm{m}$, the sampling rate was $380 \mathrm{fps}$, and the exposure time was $800 \mu \mathrm{s}$. A series of 1000 successive images were captured by the camera for blood flow video and ATFV measurement. The acquired sequence images were transmitted to the computer at a high speed. The experiment was performed in a dark room, with the system fixed to a vibration isolation optical platform. During the experiment, the animal was carefully handled in accordance with the laboratory animal protocol approved by the Institutional Animal Care and Use Committee of Foshan University.

\section{Imaging Method}

Under illumination by a low-coherence light source with a central wavelength of $540 \mathrm{~nm}$, the absorption coefficient of RBCs $\left(27.35 \mathrm{~cm}^{-1}\right)^{12}$ is much higher than that of background, such as water $\left(4.5 \times 10^{-4} \mathrm{~cm}^{-1}\right)^{13}$ and fat $\left(8.96 \times 10^{-3} \mathrm{~cm}^{-1}\right),{ }^{14}$ as shown in Fig. 1(b). An endogenous instantaneous intensity fluctuation is induced when the RBCs discontinuously traverse the blood vessel. The temporal reflected light intensity recorded by camera pixel becomes a high-frequency signal, which is the motion signal of RBCs $\left(I_{A C}\right)$. However, the background tissue only generates a direct-current signal $\left(I_{D C}\right)$ because it has no any endogenous instantaneous intensity fluctuation. This physical processing is the AIFM effect. This special phenomenon was utilized to achieve accurate ATFV measurement. It was first necessary to separate the motion signal of the RBCs from that of the background tissue. The difference between the absorption capacities of the two materials generated a fluctuating high-frequency intensity signal. The motion signal of RBCs $\left(I_{A C}\right)$ and the background signal $\left(I_{D C}\right)$ were separated in the frequency domain, as shown in Fig. 1(c). The raw temporal signal was transferred from the time $(t)$ domain to the frequency $(f)$ domain by fast Fourier transform.

The absorption capacities of the RBCs and background tissue were then obtained by high-pass and low-pass filtering, respectively. The employed formula is as follows: ${ }^{15-17}$

$$
\begin{aligned}
& I_{A C}(x, y, t)=\operatorname{HPF}[I(x, y, f)], \\
& I_{D C}(x, y, t)=\operatorname{LPF}[I(x, y, f)],
\end{aligned}
$$

where LPF[] and HPF[] denote low-pass and high-pass filtering, respectively. The recorded optical intensity can be seen to correspond to the scattering numbers, that is, $I_{D C} \propto n_{D C}$ and $I_{A C} \propto n_{A C}$, where $n_{D C}$ and $n_{A C}$ represent the background scatters and moving RBC concentration, respectively. The moving RBC concentration $\rho$ can be defined as

$\rho=\frac{n_{A C}}{n_{A C}+n_{D C}} \approx \frac{n_{A C}}{n_{D C}}=\frac{I_{A C}}{I_{D C}}$.

Note that the approximate equivalence in Eq. (2) can be rendered almost exact with the condition $n_{A C} \ll n_{D C}$.

To measure the ATFV along the vessel centerline and highlight the motion signal of the RBCs, the IMD sequence images (Video 2) can be obtained based on the ratio of $I_{A C}(x, y, t)$ to $I_{D C}(x, y, t)$

$\operatorname{IMD}(x, y, t)=\frac{I_{A C}(x, y, t)}{I_{D C}(x, y, t)}$.

In the current method of wide-field optical angiography, the AMD associated with the movement of the RBCs is given by ${ }^{11}$

$\operatorname{AMD}(x, y)=\frac{\bar{I}_{A C}(x, y)}{\bar{I}_{D C}(x, y)}$,

where $\bar{I}_{A C}(x, y)$ and $\bar{I}_{D C}(x, y)$ are, respectively, the averaged absolute values of the dynamic and static signals over time. The ATFV along the vessel centerline and the flow direction can be mapped onto the AMD image.

After separation of the signals of the RBCs and background tissue using Eq. (1), dynamic information about the blood microcirculation can be directly obtained from the Video generated from the IMD sequence images. The ATFV is calculated using the RBC cross-correlation information obtained from two relevant locations $A$ and $B$

$R_{A B}(\tau)=\int_{0}^{T_{0}} \operatorname{IMD}\left(x_{A}, y_{A}, t\right) \cdot \operatorname{IMD}\left(x_{B}, y_{B}, t+\tau\right) \mathrm{d} t$,

where $R_{A B}(\tau)$ is the IMD cross-correlation coefficient between $A$ and $B$, and $\tau$ is the transition time between the two correlation 


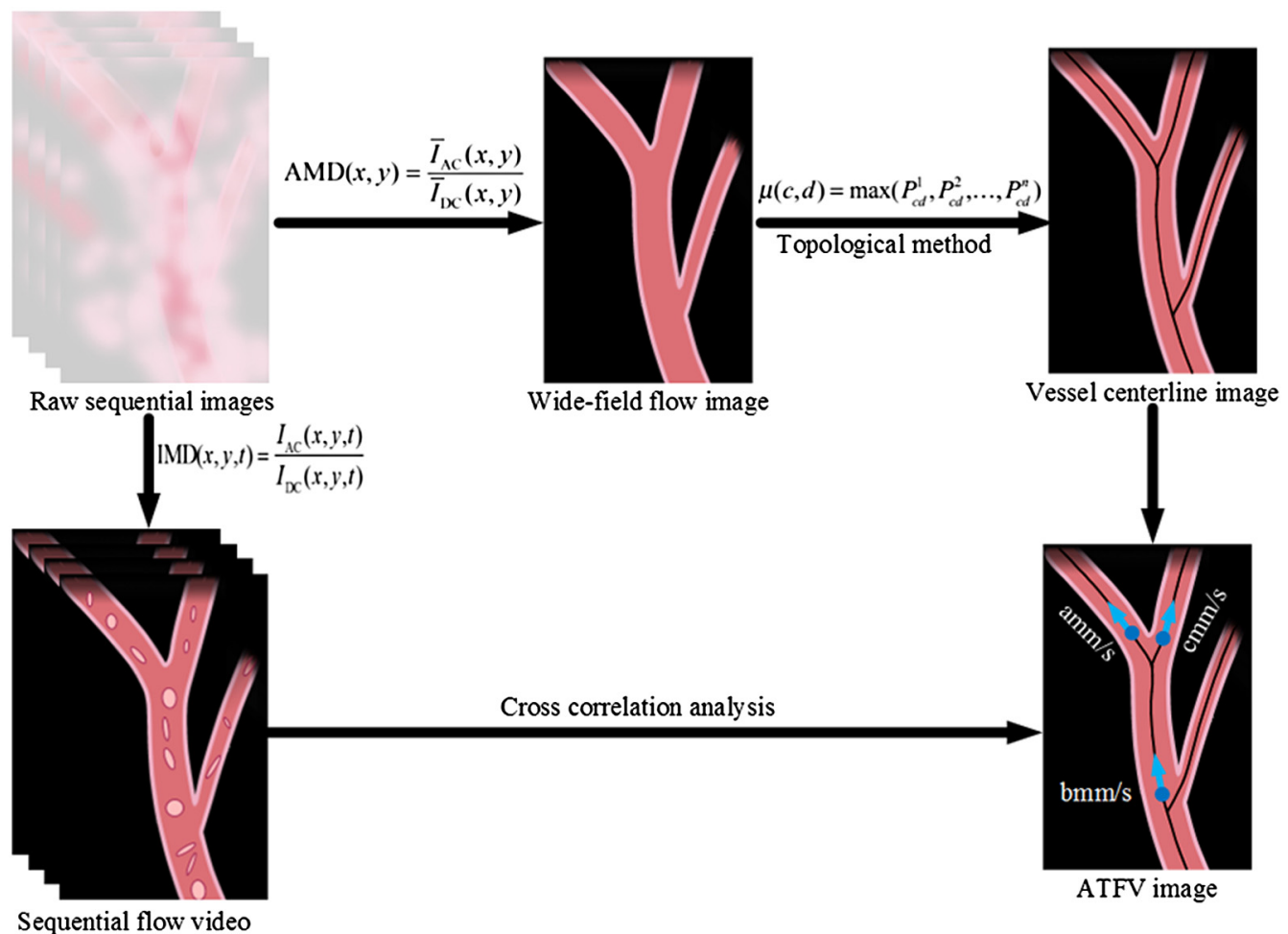

Fig. 2 The flowchart showing how ATFV along the blood vessel centerline was obtained.

locations, equals to the delay time between the highest peak of $R_{A B}(\tau)$ and the zero time. The velocity direction can be determined from the sign of $\tau_{0}$. For example, if the flow direction is from $A\left(x_{A}, y_{A}\right)$ to $B\left(x_{B}, y_{B}\right), \tau_{0}>0$; otherwise $\tau_{0}<0$. The velocity magnitude can be described as $\nu=\Delta L / \tau_{0}$. The distance between two correlation locations is defined as $\Delta L=\sqrt{\left(x_{A}-x_{B}\right)^{2}+\left(y_{A}-y_{B}\right)^{2}}$.

To obtain the binary image of the blood vessels accurately, the blood vessels must be segmented from the wide-field optical angiography image to prepare for the next step in obtaining the blood vessel centerline. An absolute fuzzy connection segmentation algorithm ${ }^{18}$ was used for this purpose in the present study. There is a fuzzy adjacency relationship between any two pixels in the image, and two concepts need to be clarified. The first is the local fuzzy relationship among the pixels, also known as the fuzzy affinity, with a value in the range 0 to 1 . It is related to the degree of spatial proximity, intensity uniformity, and intensity similarity of two adjacent pixels $(c, d)$. The other concept is the global fuzzy relationship between pixels, also known as the fuzzy connectedness, whose value is also in the range 0 to 1 . The local fuzzy relationships are used to determine the global fuzzy relationship. This involves the determination of the local fuzzy relationship value of two neighboring pixels for each path between $c$ and $d$ and adopting the lowest one as the strength of the path. The highest among all the path strength values is then determined and adopted as the global fuzzy relationship between pixels $c$ and $d$. Fuzzy adjacency can be defined as ${ }^{18}$

$\mu_{\omega}(c, d)=\left\{\begin{array}{cc}\frac{1}{1+k_{1}\left[\sqrt{\sum_{i=1}^{n}\left(c_{i}-d_{i}\right)^{2}}\right]} & \text { if } \sum_{i=1}^{n}\left|c_{i}-d_{i}\right| \leq n \\ 0 & \text { otherwise }\end{array}\right.$ where $k_{1}$ is a nonnegative constant and $n$ is the number of paths between $c$ and $d$. The affinity between any two pixels $(c, d)$ in the image can be defined as ${ }^{18}$

$\mu_{\zeta}(c, d)=\frac{\mu_{\omega}(c, d)}{1+k_{2}|f(c)-f(d)|}$,

where $\mu_{\omega}(c, d)$ is the fuzzy adjacency defined by Eq. (6). $f$ is a scene domain function, $f(c)$ and $f(d)$ are the values of the function $f$ corresponding to the pixels $c$ and $d . k_{2}$ is a nonnegative constant. The global fuzzy relationship is given by

$\mu(c, d)=\max \left(P_{c d}^{1}, P_{c d}^{2}, \ldots, P_{c d}^{n}\right)$,

where

$$
\begin{aligned}
P_{c d}^{i}= & \min \left[\mu_{\zeta}\left(c_{0}-c_{1}\right), \mu_{\zeta}\left(c_{1}-c_{2}\right), \ldots, \mu_{\zeta}\left(c_{m-1}\right.\right. \\
& \left.\left.-c_{m}\right), \mu_{\zeta}\left(c_{m}-c_{m+1}\right)\right],
\end{aligned}
$$

in which $c_{0}, c_{1}, \ldots, c_{m+1}$ are a set of pixel sequences, $c_{i}$ and $c_{i+1}$ are two adjacent pixels of their four neighboring pixels, $c_{0}=c, c_{m+1}=d$, and $n$ is the total number of paths from $c$ to $d$.

Because the vessel image is a wide-field optical angiography image, the superposition phenomenon would occur in it, resulting in a number of different vessels being mistaken as the same capillary. This would create difficulties in the image processing and analysis, and could even lead to wrong experimental results. Hence, to facilitate subsequent analysis and observation, it is necessary to distinguish between the different capillaries. The same absolute fuzzy connection segmentation algorithm is used to separate the vessels that overlap each other in the wide-field optical angiography image. 



Fig. 3 (a) Raw image (Video 1), (b) IMD image (Video 2), and (c) AMD image; and pixel values at all the points of dashed lines in the (d) raw image, (e) IMD image, and (f) AMD image. The imaged area is $2.865 \times 2.865 \mathrm{~mm}$ (Video 1, MPEG, $0.9 \mathrm{MB}$ [URL: https://doi.org/10.1117/1.JBO.23.2.026008.1]; Video 2, MPEG, 13.2 MB [URL: https://doi.org/10.1117/1.JBO.23.2.026008.2]).

After obtaining the binary image of the vessels as described above, it is necessary to refine the vessels to determine their centerlines. The topological method ${ }^{19-22}$ is a useful tool for image skeletonization, enabling the definition of pixels that can be deleted at the boundary of the image object to obtain the skeleton of the image target. However, there is the need to maintain the connectivity of the image objects without changing the number of objects in the image, the number of holes in the target, and the relationships between different objects (e.g., position relationships). Any pixel that causes a change in any of the above parameters cannot be treated as a deleted image pixel. Because the object is evenly contracted in all directions and the resulting skeleton lines are placed just at the center of the object, ${ }^{23}$ the skeleton of a vessel is considered as its centerline.

Figure 2 shows how ATFV along the blood vessels centerline was obtained. First, wide-field flow image (AMD image) and sequential flow video (IMD sequential images) were obtained according to $\operatorname{AMD}(x, y)$ and $\operatorname{IMD}(x, y, t)$, respectively. Second, vessel centerline image was then obtained based on fuzzy connection segmentation algorithm and topological method. Finally, the ATFV image was obtained by vessel centerline image and sequential flow video with cross-correlated analysis.

\section{Experimental Results}

Figure 3 shows the experimental results for a 5-day-old chicken embryo. The positions of $K$ in Figs. 3(a)-3(f) are in the same location. Figure 3(a) shows the raw image (Video 1), Fig. 3(b) shows the IMD image (Video 2), and Fig. 3(c) shows the AMD image. The difference between Figs. 3(a) (Video 1) and 3(b) (Video 2) is evident. Although the vessel contours are difficult to identify in Fig. 3(b), it contains more abundant RBC movement information. Conversely, although the vessel contours can be identified in Fig. 3(a), it is significantly less rich in RBC movement information. In Fig. 3(c), the vessels can be seen to sharply contrast with the background, with the blurry vessels in Fig. 3(a) becoming clearly visible.

Figures 3(d)-3(f) also demonstrate the effectiveness of using the AIFM effect for imaging. Figures 3(d)-3(f) show the pixel values at all the points of the dashed lines in Figs. 3(a)-3(c), respectively. As can be seen from Fig. 3(d), because of the AIFM effect, the pixel values are relatively low in the presence of the blood vessels. The pixel value is particularly significantly lower for pixels at $K$, and it is difficult to distinguish the location of the blood vessels. This is because of the higher RBC concentrations and the resultant higher light absorption. As shown in Fig. 3(e), because of the relatively low SNR of the IMD image, the signal in Fig. 3(e) is irregular. However, the RBCs are highlighted, and their movement trajectories can be clearly seen in Video 2. In Fig. 3(f), it can be seen that the signal shows a regular change, and the positions of the blood vessels can be determined according to the positions of the various peaks of the signal. For example, as can be seen at the positions of $K$ in Fig. 3(f), there are two peaks of the signal, which represent the two blood vessels at the positions of $K$ in Fig. 3(c). According to the analysis of Figs. 3(a)-3(f), the effectiveness of the AIFM effect for optical imaging is verified. 


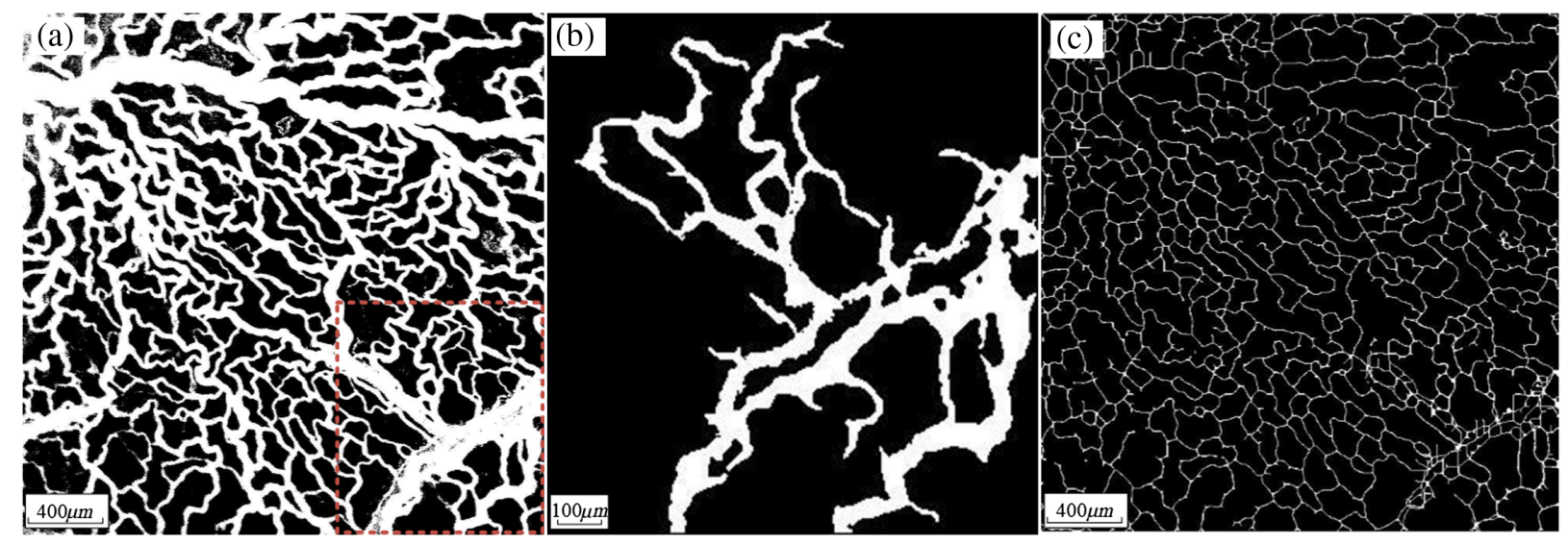

Fig. 4 (a) Binary image of chicken embryo vessels obtained by an absolute fuzzy connective segmentation algorithm, (b) binary image of the lower right part of (a) extracted by the absolute fuzzy connective segmentation algorithm, and (c) vessel centerline image determined by the topological method.

Figure 4(a) shows the binary image of the blood vessels, segmented from the AMD image using the absolute fuzzy connection algorithm. As can be seen, most of the vessels in the AMD image have been separated. Figure 4(b) shows the image of the lower right portion of Fig. 4(a) with overlapping vessels (red dashed-line box), as extracted from the AMD image using the absolute fuzzy connection segmentation algorithm. Although the algorithm cannot extract all the information about the blood vessels, the result is sufficient for distinguishing overlapping blood vessels. As can be seen from Figs. 3(c), 4(a), and 4(b), the majority of the overlapping vessels are accurately extracted. Although some vessels in the lower right portion of Fig. 4(a) cannot be extracted, it is enough to be distinguishing the overlapping blood vessels. The centerlines of the vessels are shown in Fig. 4(c), as obtained from Figs. 4(a) and 4(b) based on the topological method. As can be seen in Fig. 4(c), the centerlines of the vessels have been accurately obtained.

Figure 5 shows the results of a wide-field centerline flow transverse velocity measurement, wherein a $0.07 \mathrm{~g} / \mathrm{L} \mathrm{TiO}_{2}$ solution and glass tube of diameter $0.3 \mathrm{~mm}$ were used to simulate $\mathrm{RBC}$ movement in blood vessels. The contained $\mathrm{TiO}_{2}$ particles of diameters 8 to $12 \mu \mathrm{m}$ specifically represented the RBCs, with the $\mathrm{TiO}_{2}$ solution representing the blood flow, and the glass tube representing a blood vessel. A single-channel syringe pump was used to introduce the solution into the glass tube and control the flow rate within 0 to $8.8 \mathrm{~mm} / \mathrm{s}$. Figure 5(a) shows a raw image,
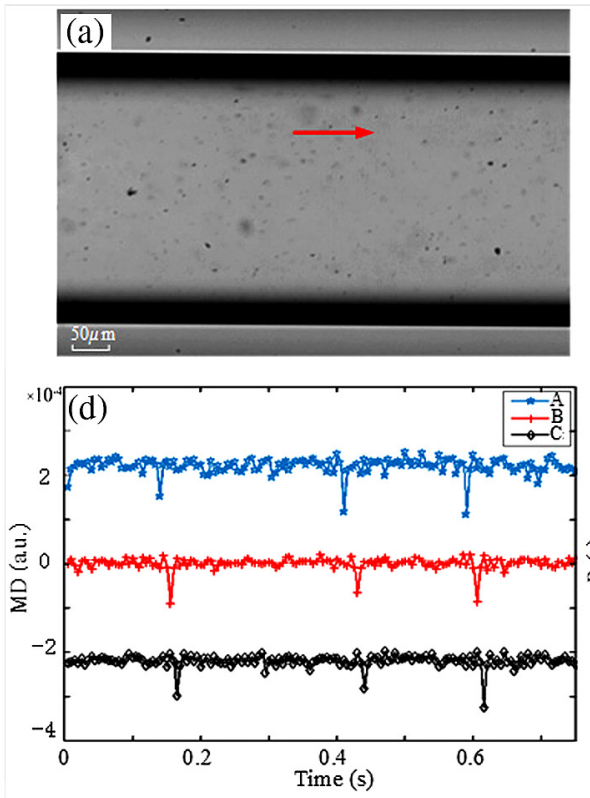
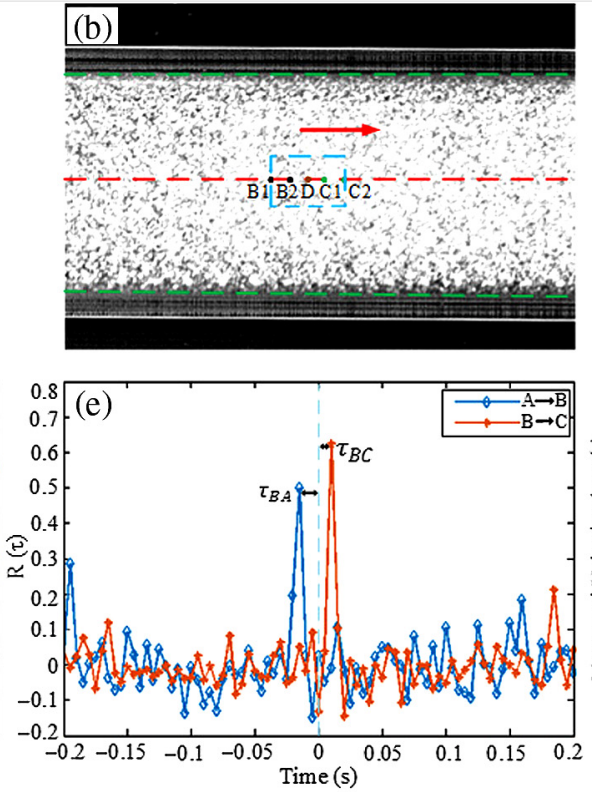
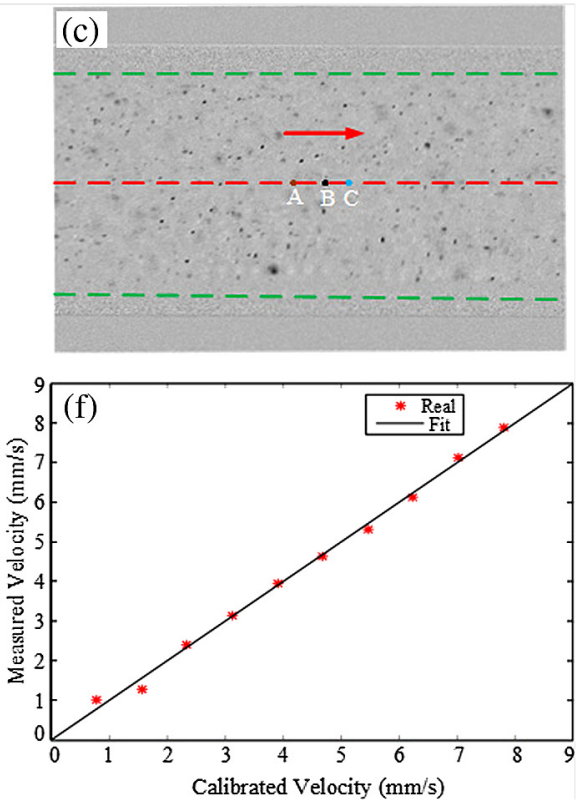

Fig. 5 (a) Raw image (Video 3), where the horizontal arrow indicates the flow direction of $\mathrm{TiO}_{2}$ solution; (b) AMD image, where $B_{1}, B_{2}, D, C_{1}$, and $C_{2}$ are different points along the centerline of the glass tube, the red dashed-line represents the centerline of the glass tube, and the green dashed-lines represent the edges of the tube; (c) IMD image (Video 4), where $A, B$, and $C$ are different points along the centerline of the glass tube, and the red and green lines are as in (b); (d) extracted IMD signals at $A, B$, and $C$; (e) values of $\tau_{B A}$ and $\tau_{B C}$ determined by the cross correlation of the IMD signals; and (f) measured and fitted velocities within 0 to $8.8 \mathrm{~mm} / \mathrm{s}$ (Video 3, MPEG, $0.68 \mathrm{MB}$ [URL: https://doi.org/10.1117/1.JBO.23.2 .026008.3]; Video 4, MPEG, 4.62 MB [URL: https://doi.org/10.1117/1.JBO.23.2.026008.4]). 

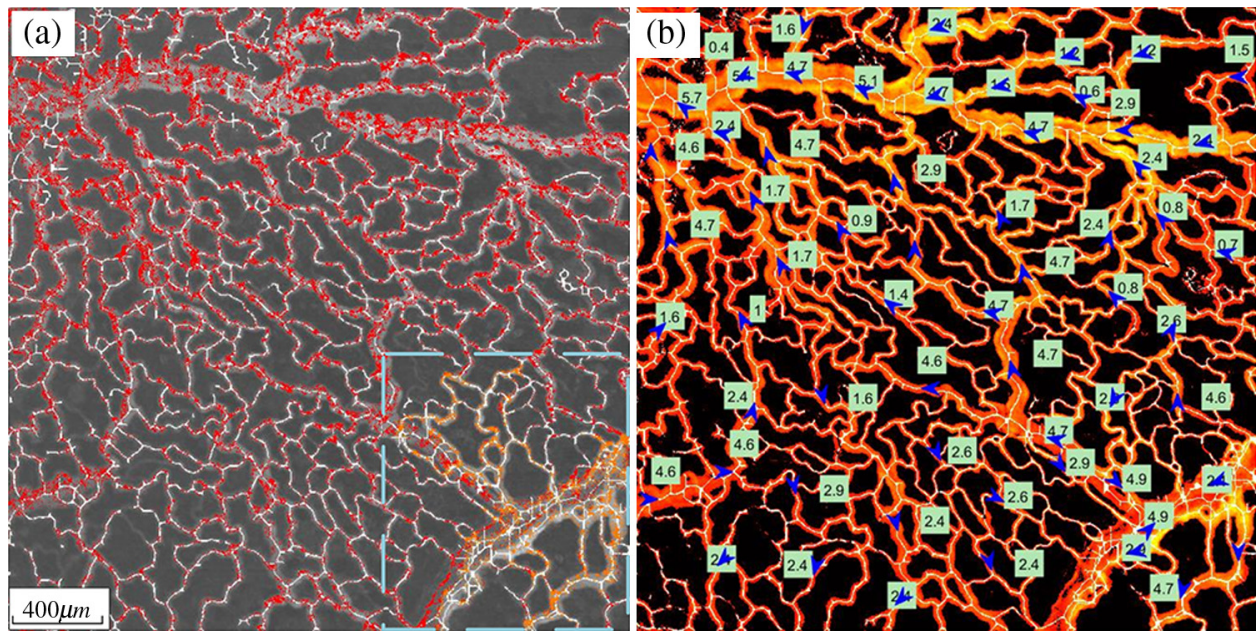

Fig. 6 (a) High SNR IMD image (Video 5) obtained using the IMD images (Video 2), the AMD image, and Figs. 4(a)-4(c); (b) wide-field centerline flow transverse blood flow velocities in the vessels of an in vivo 5day-old chicken embryo; the numbers indicate the ATFVs in $\mathrm{mm} / \mathrm{s}$, and the blue arrows indicate the flow direction. The imaged area is $2.865 \times 2.865 \mathrm{~mm}$ (Video 2, MPEG, $13.2 \mathrm{MB}$; Video 5, MPEG, $10.2 \mathrm{MB}$ [URL: https://doi.org/10.1117/1.JBO.23.2.026008.5]).

where the horizontal arrow indicates the flow direction of $\mathrm{TiO}_{2}$ solution. Figure 5(b) shows the AMD image, the cross-correlation distance is limited to the range 25 to $50 \mu \mathrm{m}$ for seeking the cross-correlation distance along the centerline in our experiment. For example, as shown in Fig. 5(b), first point $D$ was selected, and then a cross-correlation analysis was performed with the points within $\left(B_{1}, B_{2}\right)$ and $\left(C_{1}, C_{2}\right)$. The distance between $D$ and $B_{2}$ and the distance between $D$ and $C_{1}$ are each $25 \mu \mathrm{m}$. The distance between $B_{1}$ and $B_{2}$ and the distance between $C_{1}$ and $C_{2}$ are each $25 \mu \mathrm{m}$. Figure 5(c) shows the IMD image with the moving particles highlighted against the background. In Figs. 5(b) and 5(c), the red dashed-line represents the glass tube centerline, and the green dashed-lines represent the edges of the tube. Figure 5(d) shows the IMD signals extracted at points $A, B$, and $C$. The downward pulses correspond to times when a $\mathrm{TiO}_{2}$ particle appeared at each location. Figure 5(e) shows the cross-correlation profile of the IMD signals, where $\tau_{B A}$ and $\tau_{B C}$ denote the cross-correlation transition times between $B$ and $A$, and between $B$ and $C$, respectively. The flow direction is determined by the sign of the time delay. As shown in Fig. 5(e), $\tau_{B A}<0$, indicating that the RBCs flow from $A$ to $B$; and $\tau_{B C}>0$, indicating that the RBCs flow from $B$ to $C$. The particle velocity could be calculated by simply dividing the relative displacement $\Delta L$ between two correlated points (determined from their pixel numbers) by the time delay. Since the cross-correlation distance is limited to the range 25 to $50 \mu \mathrm{m}$ for seeking the cross-correlation distance along the centerline in our experiment, the cross-correlation distance is the displacement vector, and the measured velocity can be viewed as the ATFV. In Fig. 5(f), the asterisks denote the determined speeds, and the continuous line was obtained by linear fitting $\left(R^{2}=0.989\right)$. The high precision of the proposed measurement method is demonstrated by the experimental results. It should, however, be noted that, because the inner diameter of the tube is larger than that of a real blood vessel, and considering the uncertainty of the particle position, there would be some errors in the determined particle speeds.

Figure 6(a) was obtained from the IMD image (Video 2), AMD image [Fig. 3(c)], and Figs. 4(a)-4(c). Figures 4(a) and 4(b) were used as mask images for extracting the region in the IMD image that satisfied two conditions, namely a pixel value of 1 in the binary image of the chicken embryo blood vessels, and a lower pixel value in the IMD image. The AMD image and Fig. 4(c) were used as background. In Fig. 6(a), the RBCs are highlighted in red and yellow. The red and yellow RBCs that overlap move in different directions. An examination of Videos 1 and 2 in Figs. 3(a) and 3(b) and Video 5 in Fig. 6(a) reveals that the flowing RBCs in Video 1 are blurred and difficult to distinguish from the background. Although the RBCs in Video 2 are more clearly visible, the SNR is unsatisfactory. In Video 5, the movement of the RBCs in different directions is clearly more obvious, especially in the bottom right corner indicated by the blue dashed-line box.

In Fig. 6(b), the vessel centerlines of the chicken embryo accurately determined by the topological method are highlighted. The arrows in the figure indicate the flow direction, and the numbers beside the arrows indicate the determined absolute velocities $(\mathrm{mm} / \mathrm{s})$ along the vessel centerlines. The ATFVs along the vessel centerlines were calculated using the same RBC cross-correlation information at two relevant positions A and B along the centerlines, as obtained from the high-SNR IMD image. The calibration velocities indicated in Fig. 6(b) are within 0.4 to $5.7 \mathrm{~mm} / \mathrm{s}$; most of those in the smaller vessels (with diameter smaller than $50 \mu \mathrm{m}$ ) are $<3 \mathrm{~mm} / \mathrm{s}$, whereas those in the larger vessels (with diameter larger than $51 \mu \mathrm{m}$ ) are within 2.4 to $5.7 \mathrm{~mm} / \mathrm{s}$. With increasing ATFV, the RBCs in the smaller vessels flow into the larger ones. In addition, the ATFVs are relatively high where a number of vessels are gathered.

\section{Discussions and Conclusions}

Although the proposed method achieves highlighting and tracking of RBCs and enables accurate measurement of the ATFV along the vessel centerline, it is only suitable for a transparent sample and requires that the blood vessels not be too large, as in chicken embryos and zebra fish. Since our method is limited by the depth of field, it is difficult to achieve deep blood flow measurement. Therefore, we consider combining 
our method with the diffuse correlation spectroscopy (DCS) to develop the flow imaging method for the deep tissue such as the cerebral blood flow measurement proposed in related articles. ${ }^{24,25}$ In addition, the method cannot be applied to overlapping vessels because it considers the transverse speed. In the present study, the maximum value of the cross-correlation profile of the IMD signals was used as the cross-correlation peak to determine the cross-correlation distance and compute the delay time. However, when RBCs flow through a large vessel in a turbid tissue, individual RBCs form RBC clusters, and their shape, direction, and separation distance are varied and uncertain. This will cause a lower-SNR IMD signal. In this case, the present method for determining the cross-correlation peak is not precise. Thus, further study is required to determine some constraints for choosing the cross-correlation peak.

In conclusion, we investigated the wide-field optical angiography image of a living specimen and successfully highlighted the RBCs' signal against the background tissue and determined the ATFV along the blood vessel centerline. This method can help to provide technical reference for the research on the role of blood flow velocity in the pathogenesis of anemia, thrombosis, retinopathy, and other vascular diseases.

\section{Disclosures}

The authors have no relevant financial interests in this article and no potential conflicts of interest to disclose.

\section{Acknowledgments}

This work was supported by National Natural Science Foundation of China $(61771139,11474053,61471123,61605026$, and 81601534), Science and Technology Planning Project of Guangdong Province (2016A020220021), Natural Science Foundation of Guangdong Province (2015A030313639), Central of China Supporting Local Finance Project, Special Fund for Science and Technology Innovation of Foshan (2015GA10001), Foshan Science and Technology Type for Small and Mediumsized Enterprise Technology Innovation Fund Project (2014AA100802), and University Key Project of Guangdong Province (2015xzd02).

\section{References}

1. C. E. Riva, A. Alm, and C. J. Pournaras, Ocular Circulation, Elsevier, Amsterdam (2012).

2. M. Cipolla, The Cerebral Circulation, Morgan and Claypool Life Sciences, California (2009).

3. J. D. Briers and S. Webster, "Laser speckle contrast analysis (LASCA): a nonscanning, full-field technique for monitoring capillary blood flow," J. Biomed. Opt. 1, 174-179 (1996).

4. A. Nadort et al., "Quantitative blood flow velocity imaging using laser speckle flowmetry," Sci. Rep. 6, 25258 (2016).

5. M. Leutenegger et al., "Real-time full field laser Doppler imaging," Biomed. Opt. Express 2, 1470-1477 (2011).

6. C. E. Riva et al., "Bidirectional LDV system for absolute measurement of retinal blood speed," Appl. Opt. 18, 2301-2306 (1979).

7. M. Nagahara et al., "In vivo measurement of blood velocity in human major retinal vessels using the laser speckle method," Invest. Ophthalmol. Visual Sci. 52, 87-92 (2011).

8. A. Yoshida et al., "Reproducibility and clinical application of a newly developed stabilized retinal laser Doppler instrument," Am. J. Ophthalmol. 135, 356-361 (2003).

9. A. Guevaratorres, A. Joseph, and J. Schallek, "Label free measurement of retinal blood cell flux, velocity, hematocrit and capillary width in the living mouse eye," Biomed. Opt. Express 7, 4228-4249 (2016).
10. C. P. Kothandaraman and R. Rudramoorthy, Fluid Mechanics and Machinery, New Age International (P) Ltd., New Delhi (2007).

11. Y. Zeng et al., "Laser speckle imaging based on intensity fluctuation modulation," Opt. Lett. 38, 1313-1315 (2013).

12. S. S. Ulyanov and V. V. Tuchin, "Use of low-coherence speckled speckles for bioflow measurements," Appl. Opt. 39, 6385-6389 (2000).

13. G. M. Hale and M. R. Querry, "Optical constants of water in the $200 \mathrm{~nm}$ to $200 \mathrm{~mm}$ wavelength region," Appl. Opt. 12, 555-563 (1973).

14. R. Veen et al., "Determination of VIS-NIR absorption coefficients of mammalian fat, with time- and spatially resolved diffuse reflectance and transmission spectroscopy," in OSA Annиal Biomedical Topical Meeting, Paper SF4 (2004).

15. R. K. Wang et al., "Three dimensional optical angiography," Opt. Express 15, 4083-4097 (2007).

16. Y. K. Tao, A. M. Davis, and J. A. Izatt, "Single-pass volumetric bidirectional blood flow imaging spectral domain optical coherence tomography using a modified Hilbert transform," Opt. Express 16, 1235012361 (2008)

17. Y. K. Tao, K. M. Kennedy, and J. A. Izatt, "Velocity-resolved 3D retinal microvessel imaging using single-pass flow imaging spectral domain optical coherence tomography," Opt. Express 17, 4177-4188 (2009).

18. J. K. Udupa and S. Samarasekera, "Fuzzy connectedness and object definition: theory, algorithms and applications in image segmentation," Graphical Models Image Process. 58, 246-261 (1996).

19. R. M. Haralick and L. G. Shapiro, Computer and Robot Vision, Addison-Wesley, New Jersey (1992).

20. T. Y. Kong and A. Rosenfeld, Topological Algorithms for Digital Image Processing, Elsevier Science Inc., New York (1996).

21. L. Lam, S. W. Lee, and C. Y. Suen, "Thinning methodologies-a comprehensive survey," IEEE Trans. Pattern Anal. Mach. Intell. 14, 869885 (1992).

22. W. K. Pratt, Digital Image Processing, John Wiley and Sons, Inc., New York (1991).

23. M. Sonka, V. Hlavac, and R. Boyle, Image Processing, Analysis, and Machine Vision, Cengage Learning, Boston (2014).

24. Y. Shang, T. Li, and G. Yu, "Clinical applications of near-infrared diffuse correlation spectroscopy and tomography for tissue blood flow monitoring and imaging," Physiol. Meas. 38, R1-R26 (2017).

25. D. Turgut and A. G. Yodh, "Diffuse correlation spectroscopy for noninvasive, micro-vascular cerebral blood flow measurement," Neuroimage 85, 51-63 (2014).

Nanshou Wu is a second-year graduate student at Foshan University in Foshan, China. He received his bachelor's degree in mechanical and electronic engineering from Guangzhou College of South China University of Technology. His current interest is in precision optical detection technology and image processing.

Mingyi Wang is an assistant professor at Foshan University and she earned her bachelor's degree at Beijing Normal University, where she completed her PhD in 2016. Currently, she is engaged in optical imaging and optical testing.

Dingan Han is a professor at Foshan University and she received her bachelor's degree from Hunan Normal University and PhD at South China Normal University in 2005. Her current research interests include biological optics imaging and computer applications.

Haishu Tan is a professor at Foshan University. He received his bachelor's degree in electronic technology and his $\mathrm{PhD}$ in optical engineering from Tianjin University, in 1994 and 1998, respectively. Currently, he is studying three-dimensional optical measurement and photoelectric detection.

Yaguang Zeng is an associate professor at Foshan University and the head at Foshan Laser Special System and Equipment Engineering Technology Research Center. He received his PhD in electronic technology in 2014 at Beijing Normal University. Currently, he is studying optical imaging and optical testing.

Biographies for the other authors are not available. 